\title{
A Positive-Working Alkaline Developable Photosensitive Polyimide for Second-Order Nonlinear Optics
}

\author{
Yoshimasa Sakai, Mitsuru Ueda*, Takashi Fukuda ${ }^{\dagger}$ and Hiro Matsuda ${ }^{\dagger}$ \\ *Depertment of Human Sensing and Functional Sensor Engineering, Graduate School of Engineering, \\ Yamagata University, Yonrzawa, Yamagata 992-8510 Japan and ${ }^{\dagger}$ National Insitute of Materials and \\ Chemical Research, 1-1 Higashi, Tsukuba, Ibaraki, 305-8565 Japan
}

\begin{abstract}
A poly(amic acid) having second-order nonlinear optical (NLO) properties was preparedby the ring-opening pol yaddition of pyrom el li tic dianhydri de (PMDA) with 5,5'-(hex afluoroi sopropyl idene)bis $\{2-[2-[N$-ethyl- $N-(4-(4-n i$ trophenyldiazenyl)phenyl)aminoethoxy]aniline]\} (HNDA) and bis(4-aminophenyl) ether (BAPE). The polymer containing $30 \mathrm{wt} \%$ of 2,3,4,-tris [1-oxo-2-diazonaphthoquinone-4-sulfon yl ox y] benzophenone (D4SB) as a photosensitive dissolution inhibitor functioned as an alkal ine developable photosensitive resist, and then was converted to pol yi mi de by corona pol ing during the thermal i mid zation process. The second harmoni c coefficient $\left(d_{33}\right)$ of the resul ting pol yi mide at the wavel en gth of 1064 $\mathrm{nm}$ was $53 \mathrm{pm} / \mathrm{V}\left(127.3 \times 10^{-9} \mathrm{esu}\right)$ and rem ai ned unchanged at elevatedtem peratures.

Keywords: Photosensitive polyimide, Second-order nonlinear optical property, Corona poling, Second harmonic coefficient,
\end{abstract}

\section{Introduction}

Second-order nonlinear optical (NLO) materials are currently of interest to a larger number of research groups as they have potential applications in area such as telecommunications, optical information processing and data storage. [1] One of the major difficulty with these materials is the long term relaxation of the NLO chromophores back to the isotoropic state. Therefore, recent research efforts have been focused on enhancing the stability of dipole orientation at elevated temperatures.[2a-e] Polyimides (PIs) are excellent candi-dates for electro-optical materials because of low dielectric constants and high glass transition temperatures $\left(T_{g}\right)$, and are compatible with semiconductor processes.

On the other hand, photosensitive polyimides (PSPIs) are currently receiving considerable attention for their potential use in the fabrification of semiconductor devices and multichip modules, since they enable the number of process steps to be reduced by avoiding the use of classical photo- resists. [3]

In a preceding paper,[4] we reported a new positive-working alkaline developable PSPI based on poly(amic acid) containing a nonlinear optical chromophore and 2,3,4,-tris[1-oxo-2-diazonaphthoquinone-5-sulfonyloxy]benzophenone

(D5SB). This is consistent with the trend of using aqueous base solutions as developers for the photoresist processing technology in the microelectronics industry. We also reported that polyimides derived from diamine with two Disperse Red-1 units showed the large second harmanic coefficient $\left(d_{33}=116 \mathrm{pm} / \mathrm{N}\right)$ at the wavelength of $1064 \mathrm{~nm}$ and the value remained unchanged at elevated temperature. [5] Therefore, the synthesis of PSPI with NLO properties will be interested in developing effective electro-optical devices.

We now reports the new positive working alkaline developable PSPI having second-order NLO properties. 


\section{Experimental}

\subsection{Materials.}

$\mathrm{N}$-Methyl-2-pyrrolidinone (NMP), $\quad \mathrm{N}, \mathrm{N}$ dimethylacetamide (DMAc), and $N, N$-dimethyl formamide (DMF) were stirred over powdered calcium hydride overnight and then distilled under reduced pressure and stored over 4A molecular sieves. Tetrahydrofuran (THF) was purified by distillation over sodium chips and benzophenone ketyl. Phthalic anhydride and pyromellitic dianhydride (PMDA) were recrystallized from acetic anhydride. Bis(4aminophenyl) ether (BAPE) was recrystallized from isopropyl alcohol. Other reagents and solvents were obtained commercially and were used as received.

\subsection{Monomer Synthesis.}

$5,5^{\prime}$-(Hexafluoroisopropylidene)bis $\{2-[2-[N-$ ethyl- $\mathrm{N}$-(4-(4-nitrophenyldiazenyl)phenyl)aminoethoxy]aniline]\} (HNDA) was prepared by Mitsunobu reaction, according to the reported procedure.[5] The yield was $60 \%: \mathrm{mp}=176-178$ ${ }^{\circ} \mathrm{C}$; IR (KBr) : v $3420(\mathrm{~N}-\mathrm{H}), 1330 \mathrm{~cm}^{-1}\left(-\mathrm{NO}_{2}\right)$. ${ }^{1} \mathrm{H}-\mathrm{NMR}$ (DMSO-d ${ }_{6}$ ) d 8.6-6.4 (m,PhH, 22H), 4.9 $\left(\mathrm{s}, \mathrm{NH}_{2}, 4 \mathrm{H}\right), 4.2-3.6\left(\mathrm{t},-\mathrm{CH}_{2}-, 12 \mathrm{H}\right), 1.2\left(\mathrm{t}, \mathrm{CH}_{3}\right.$, 6H). Anal. Calcd for $\mathrm{C}_{47} \mathrm{H}_{44} \mathrm{~F}_{6} \mathrm{~N}_{10} \mathrm{O}_{6}: \mathrm{C}, 58.87 ; \mathrm{H}$, 4.62; N, 14.61. Found : C, 58.79; H, 4.69; N, 14.59.

\subsection{Preparation of Poly(amic acid).}

A solution of HNDA $(0.96 \mathrm{~g}, 1.0 \mathrm{mmol})$ and BAPE $(0.20 \mathrm{~g}, 1.0 \mathrm{mmol})$ in NMP $(4.6 \mathrm{~mL})$ was cooled with an ice-water bath. To this solution was added with stirring PMDA (0.44 g, $2.0 \mathrm{mmol})$. The mixture was stirred at room temperature for 6 $h$. The resulting viscous solution was diluted with NMP $(3.5 \mathrm{~mL})$ and poured into a $50 \%$ aqueous methanol solution. The poly(amic-acid) that precipitated was filtered off and dried in vacuo at $50^{\circ} \mathrm{C}$ for $12 \mathrm{~h}$. The yield was $1.47 \mathrm{~g}(92 \%)$. The inherent viscosity of the polymer in NMP was 0.35 $\mathrm{dL} / \mathrm{g}$ at a concentration of $0.5 \mathrm{~g} / \mathrm{dL}$ at $30^{\circ} \mathrm{C}$. IR $(\mathrm{KBr})$ : v $3420(\mathrm{~N}-\mathrm{H}), 1720,1660(\mathrm{C}=\mathrm{O}), 1330$ $\mathrm{cm}^{-1}\left(-\mathrm{NO}_{2}\right)$. Anal.Calcd for $\mathrm{C}_{79} \mathrm{H}_{60} \mathrm{~F}_{6} \mathrm{~N}_{12} \mathrm{O}_{19} \cdot 3.2$ $\mathrm{H}_{2} \mathrm{O}$ : C,57.51 \%; H,4.13\%; N,10.19 \%. Found: C,57.52 \%; H,4.19\%; N,10.25\%.

\subsection{Photosensitivity.}

Polymer was dissolved in $20 \mathrm{wt} \%$ in DMAc.at room temperature, and to this solution was added D4SB. Films spin-cast on silicone wafers were prebaked at $80^{\circ} \mathrm{C}$ for $10 \mathrm{~min}$ and exposed through a super-high-pressure mercury lamp (Ushio USH-200DP). Imagewise exposure through a mask was carried out in a contact-printing mode. The film thickness was measured by Dektak 3030 system (Veeco Instruments Inc.).

\subsection{Corona Poling of Poly(amic acid) films.}

Polymer was dissolved in $20 \mathrm{wt} \%$ in DMAc at room temperature. Films spin-cast on glass substrate were prebaked at $80^{\circ} \mathrm{C}$ for $10 \mathrm{~min}$. The average film thickness was $0.4 \mu \mathrm{m}$. The corona poling of the NLO substituted poly(amic acid) (PAA) to the corresponding polyimide (PI) was carried out the following two conditions.

(i) PI-1: Poling during imidization. The PAA film was heated at a rate of $10^{\circ} \mathrm{C} / \mathrm{min}$ from room temperature to a specified temperature and kept at this temperature for $1 \mathrm{~h}$ under air or $\mathrm{N}_{2}$ with $5.0 \mathrm{kV}$ applied to the discharging needle. The sample poled was cooled to room temperature, and the $\mathrm{DC}$ field was then removed.

(ii) PI-2: Poling after imidization. The PAA film was heated at a rate of $10^{\circ} \mathrm{C} / \mathrm{min}$ from room temperature to $250^{\circ} \mathrm{C}$, and kept at this temperature for $1 \mathrm{~h}$. The poling field was applied and the sample was heated a specified temperature in the range of 100 to $280^{\circ} \mathrm{C}$ for $1 \mathrm{~h}$ under air or $\mathrm{N}_{2}$.

\subsection{Measurements.}

The infrared spectra were recorded on a HORIBA FT-210 spectrometer. The UV-visible spectra were obtained on a JASCO V-560 spectrophotometer. Thermogravimetric analyses (TG) were performed on a Seiko SSCS200 (TG/DTA 220) thermal analyzer at a heating rate of $10^{\circ} \mathrm{C} / \mathrm{min}$. The glass trnasition temperature was measured on a Seiko SSS 5000 DSC 220 differential calorimetry (DSC) at a heating rate of $20^{\circ} \mathrm{C} /$ min under nitrogen. Molecular weights were determined by gel permeation chromatography (GPC) with polystyrene standard using a JASCO HPLC system eqquiped with a Shodex KD-80M column at $40{ }^{\circ} \mathrm{C}$ in DMF containing $10 \mathrm{mmol} / \mathrm{L}$ LiBr. Nonlinear optical measurements were performed using a standard Maker Fringe technique. The second harmonic signal generated by the fundamental wave $(1064 \mathrm{~nm})$ was detected on a YG 582 special Actively Q-Switched Nd:YAG Laser;Quantel International and TDL 50 Nd:YAG Pumped Dye Laser;Quantel International. After amplification it was average in a boxcar integrator. A quartz crystal was used as the reference sample. 


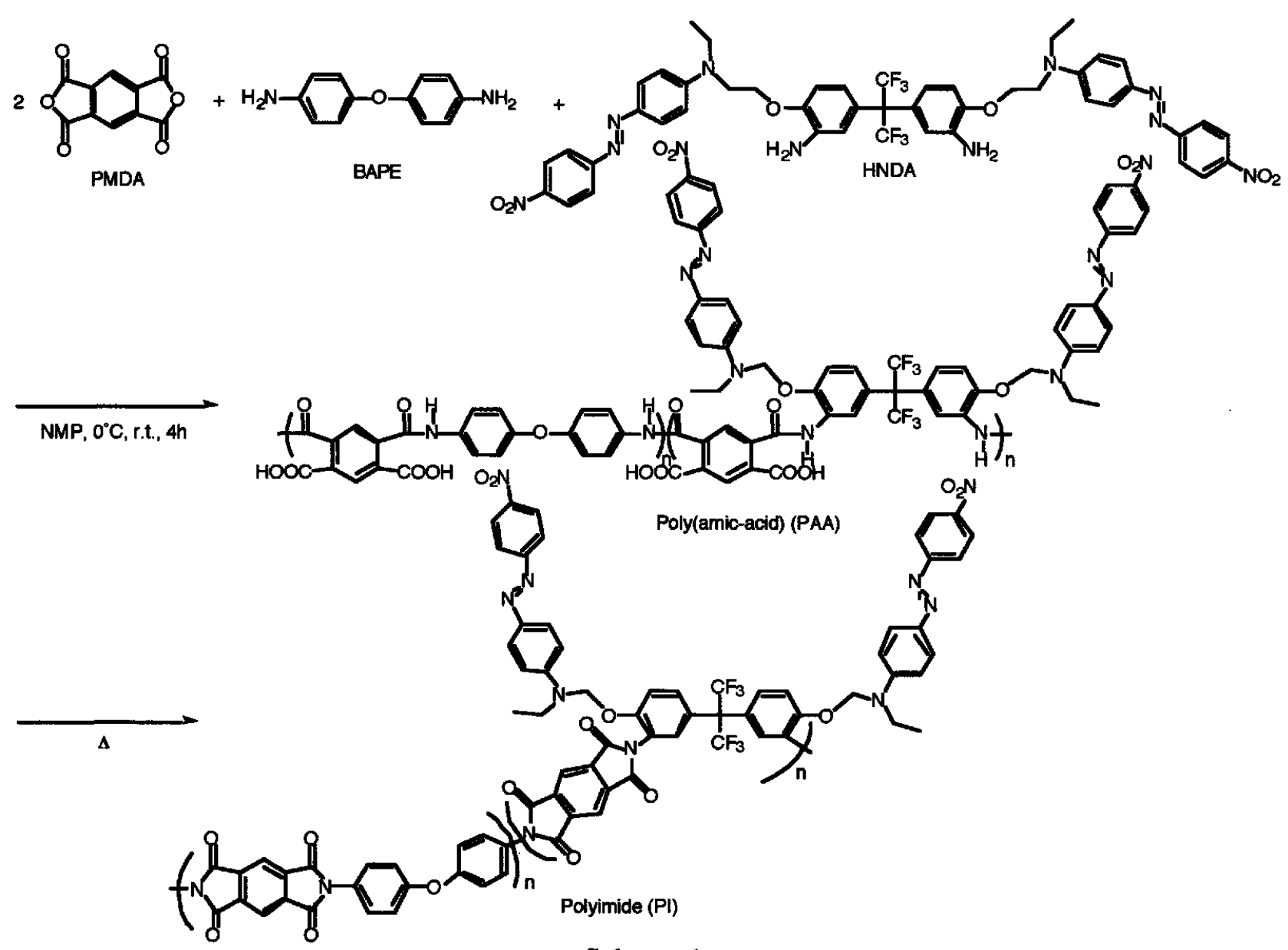

Scheme 1.

\section{Results and Discussion}

\subsection{Synthesis of Poly(amic acid).}

Considering the solubility and film properties of the resulting poly(amic acid) (PAA) and polyimide (PI), we decided to use bis(4-aminophenyl) ether (BAPE) as a co-diamine. The ringopening polyaddition of pyromellitic dianhydride (PMDA) with $5,5^{\prime}$-(hexafluoroisopropylidene)bis $\{2-[2-[N$-ethyl- $N$-(4-(4-nitrophenyldiazenyl)phenyl)aminoethoxy]aniline]\}

(HNDA) and BAPE was carried out in NMP at room temperature and proceeded smoothly to produce the PAA with an inherent viscosity of $0.35 \mathrm{dL} / \mathrm{g}$ (Scheme 1.).

The structure of PAA was characterized by infrared spectroscopy and elemental analysis. The IR spectrum showed characteristic absorptions at 1720 and $1660 \mathrm{~cm}^{-1}$ due to the carbonyl groups of carboxylic acids and amides, respectively. Elemental analysis also supported the formation of the expected polymer.

The PAA was a reddish purple powder, soluble in polar aprotic solvents such as NMP and DMAc at room temperature, and insoluble in common organic solvents. A transparent film was cast from the solution of the PAA in DMAc. The molecular weight of the polymer having an inherent viscosity of $0.35 \mathrm{dL} / \mathrm{g}$ was determined by GPC. The chromatograph indicated that the relative $\mathrm{Mn}$ and Mw values were 25,000 and 68,000 , respectively, relative to polystyrene standards. The $\mathrm{Mw} / \mathrm{Mn}$ was 2.7.

\subsection{Lithographic Evalution.}

The selection of a photoactive compound is very important for the accomplishment of a good pattern image. The UV-visible spectrum of $1 \mu \mathrm{m}$ thick film of the PAA indicates that the film has a strong absorption centered at $500 \mathrm{~nm}$ due to Disperse Red-1 and is relatively transparent around $370 \mathrm{~nm}$. (Figure 1.) Thus, we chose 2,3,4,-tris[1-oxo-2-diazonaphthoquinone-4-sulfonyloxy]benzophenone (D4SB) as a photosensitive $o$-diazonaphthoquinone (DNQ) compound which has a strong absorption in the range of $300-450 \mathrm{~nm}$.

After preliminary optimization studies involving D4SB loading and a concentration of 


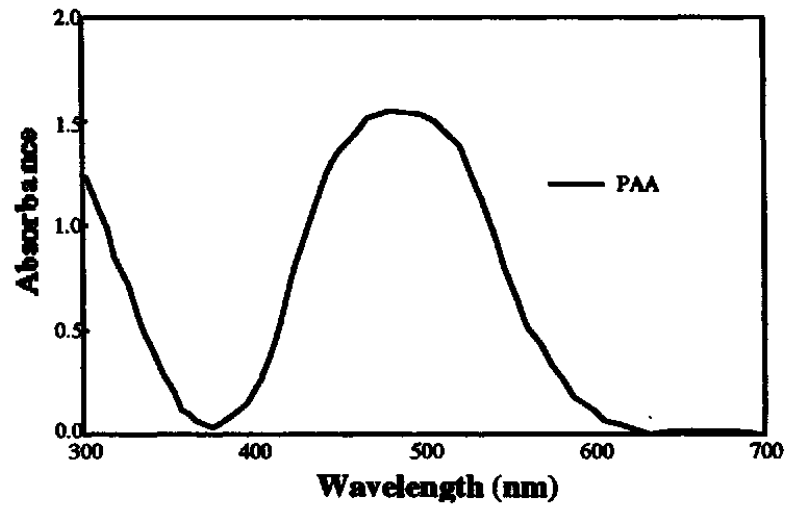

Figure 1. UV-visible spectra of the PAA.

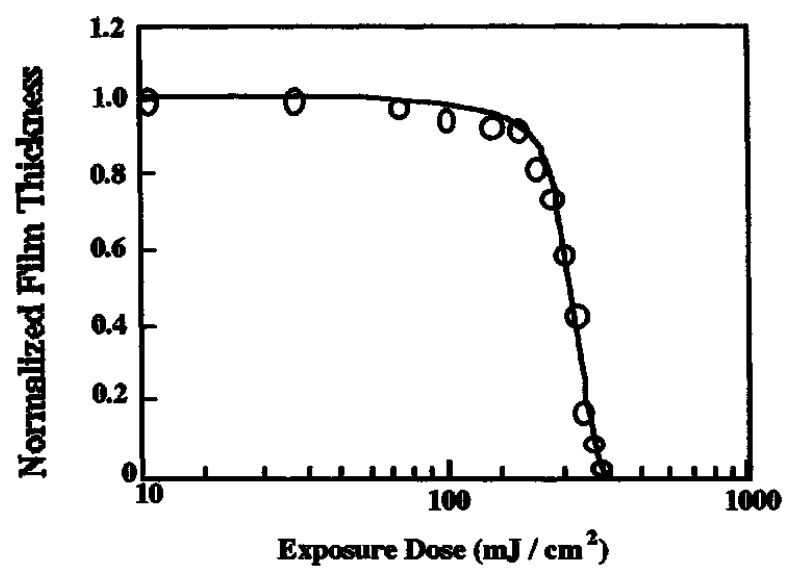

Figure 2. Exposure characteristic curve of the system based on PAA (70 wt\%) and D4SB (30 wt\%), using the $0.5 \mathrm{wt} \%$ aqueous TMAH solution as a developer.

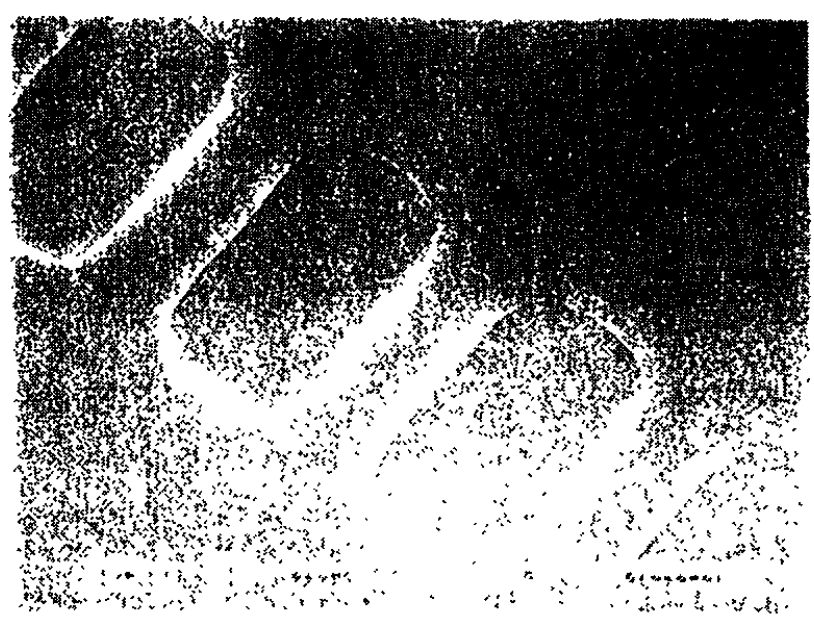

Figure 3. A scanning electron micrograph of positive imageprinted in PAA film.

aqueous tetramethylammonium hydroxide (TMAH) solution, we prepared a photosensitive system consisting of the PAA (70 wt\%) and D4SB (30 wt\%). The sensitivity curves for $1 \mu \mathrm{m}$ thick film of the PAA, shown in Figure 2, indicates that the sensitivity and contrast were $400 \mathrm{~mJ} / \mathrm{cm}^{2}$ and
3.2 with $365 \mathrm{~nm}$-light, respectively, after development with a $0.5 \%$ aqueous TMAH solution at room temperature.

In Figure 3 is presented a scanning electron micrograph of the contact-printed image that was obtained using a resist composed of PAA $(70 \mathrm{wt} \%)$ and D4SB (30 wt\%), postbaked at at $140{ }^{\circ} \mathrm{C}$ for 10 min after exposure to $400 \mathrm{~mJ} / \mathrm{cm}^{2}$ and developing with the 0.5 TMAH aqueous solution. The resist is capable of resolving $8 \mu \mathrm{m}$ features when $1 \mu \mathrm{m}$ thick films are used.

\subsection{Thermal Properties of the Polymer.}

The efficiency and stability of the dipole orientation of the resulting polyimide (PI) are influenced by several factors such as a imidization temperature of PAA to PI, a thermal stability of NLO chromophore and a glass transition temperature ( $\left.\mathrm{T}_{2}\right)$ of PI. Thus, thermal properties of the PAA was examined by thermogravimetry (TG) and differential scanning calorimetry (DSC). Figure 4 showed TG curves of the PAA film and the film cured at $250^{\circ} \mathrm{C}$ for $1 \mathrm{~h}$. The weight loss due to the elimination of absorbed water and the imide formation started at $120^{\circ} \mathrm{C}$ and continued until around $225^{\circ} \mathrm{C}$ in nitrogen. The second weight loss observed around $280^{\circ} \mathrm{C}$ is caused by the decomposition of chromophores. The last stage of weight loss appeared at about $500{ }^{\circ} \mathrm{C}$. This feature is attributed to degradation of the main chain. On the other hand, the polymer cured at $250{ }^{\circ} \mathrm{C}$ for $1 \mathrm{~h}$ showed no weight loss up to 280 ${ }^{\circ} \mathrm{C}$. These results indicate NLO chromophores in polymer are stable at high temperature.

Thermal imidization process of the PAA was followed by IR spectroscopy. The PAA film was heated at a rate of $10^{\circ} \mathrm{C} / \mathrm{min}$ from room temperature to a specified temperature $(160,200$ and $250^{\circ} \mathrm{C}$ ) and kept at this temperature for $1 \mathrm{~h}$.

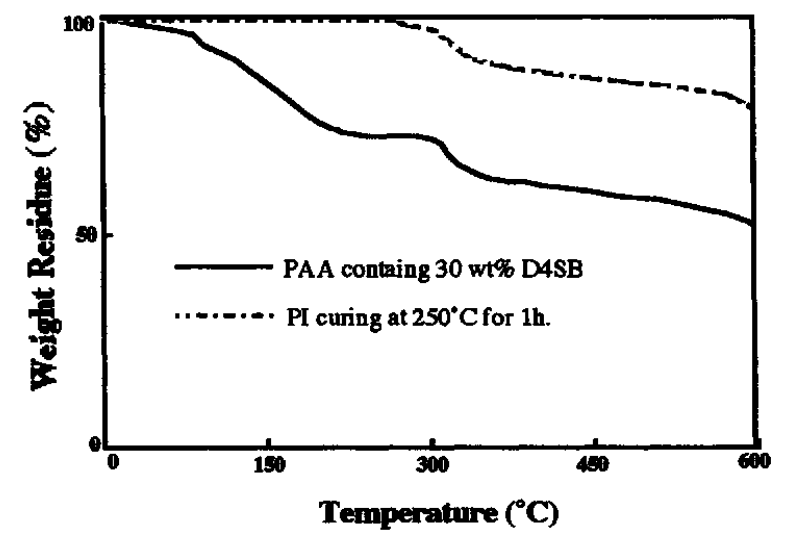

Figure 4. TG curves of PAA and PI films. 
The IR spectra of thermally treatedPAAfilm at each temperature, shown in Figure 5, indicate the imidization was almost completed at $200^{\circ} \mathrm{C}$, where characteristic imide absorptions at 1780 and 1720 $\mathrm{cm}^{-1}$ were observed. The glass transition temperature $\left(T_{g}\right)$ of $P I$ was observed at $202^{\circ} \mathrm{C}$ in DSC curve. This $T_{g}$ is quite adequate to meet the imidization reaction without decomposition of NLO chromophore, dipole alignment of the NLO polyimide, and the stabilization of NLO chromophore alignment.

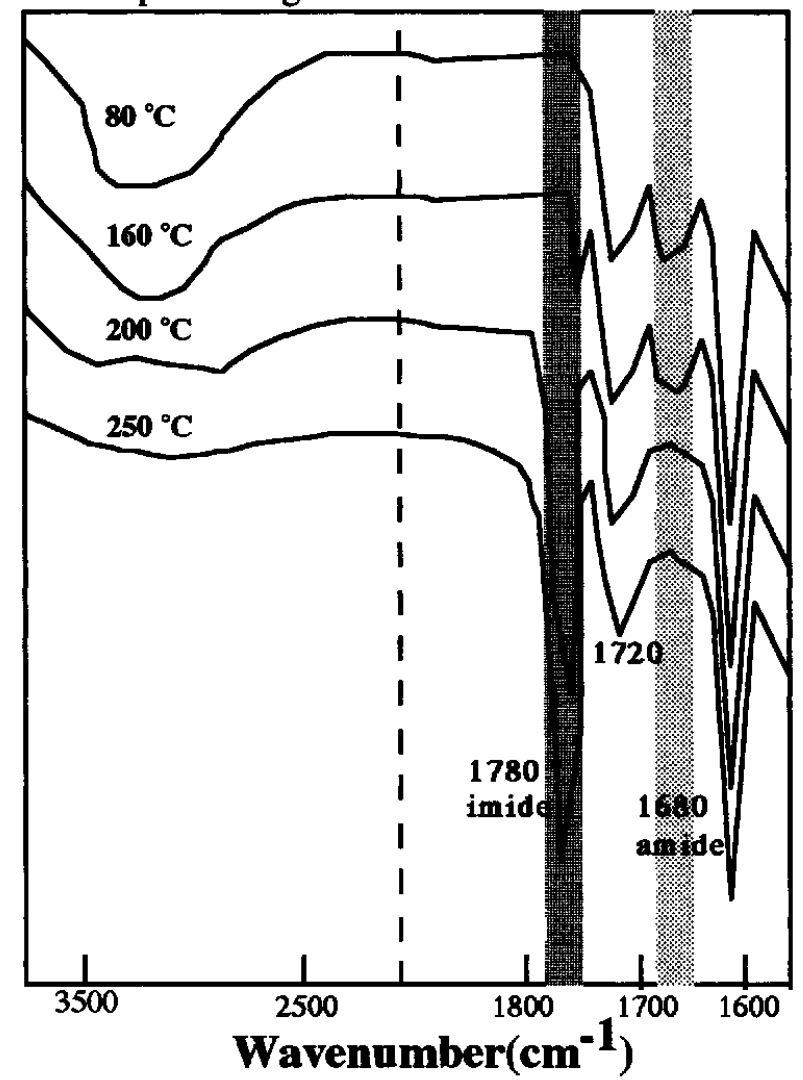

Figure 5. Change of IR spectra of the PAA on thermal treatment.

\subsection{Nonlinear Optical Properties.}

During the corona poling process accompanying imidization, a second harmonic generation (SHG) of PAAs doped with NLO chromophores generally decreases to zero, reappears, and increases with increasing temperature.[6] On the other hand, the SHG intensity for poling of polyimides increases monotonically with increasing temperature.[6] The orientational process of the chromophore in the corona poling by measuring the SHG from the polymer was studied. The SHG intensity was measured by the following two conditions; (i) PI-1: Poling during imidization, (ii) PI-2: Poling after imidization. under air or $\mathrm{N}_{2}$.

Figure 6 showed the relationship between the relative SHG intensity of the PAA film in the pol-

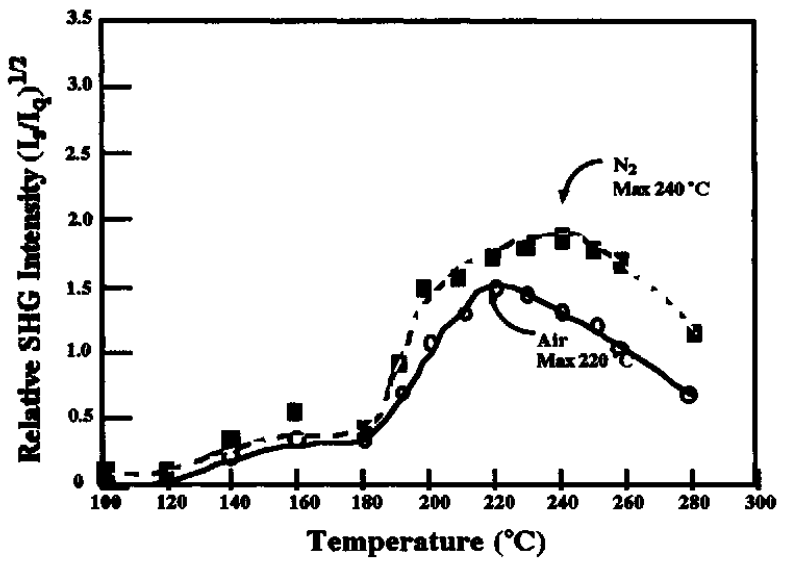

Figure 6. Relationship between the SHG intensity of PI-1 and thermally treated temperature in the corona poling.

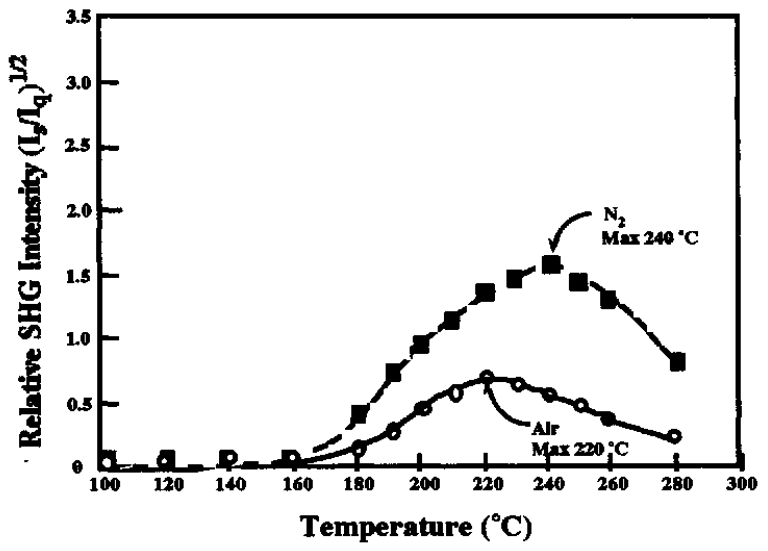

Figure 7. Relationship between the SHG intensity of PI-2 and thermally treated temperature in the corona poling.

-ing and the thermally treated temperature. The relative SHG intensities increased monotonically with increasing temperature until at $220^{\circ} \mathrm{C}$ in air and $240{ }^{\circ} \mathrm{C}$ in $\mathrm{N}_{2}$, respectively, and then decreased above these temperatures probably because of the degradation of chromophores at high temperature under high electric field. On the other hand, the relative SHG intensity of the corresponding polyimide rapidly increases over $200{ }^{\circ} \mathrm{C}$, and reaches the maximum values at $220^{\circ} \mathrm{C}$ in air and $240{ }^{\circ} \mathrm{C}$ in $\mathrm{N}_{2}$, respectively (Figure 7). These differences in SHG profiles might be attributed to the difference Tg between the PAA and the PI. The alignment of the chromophores proceeds at around the $T_{g}$ of PI and the SHG intensity increases in this temperature range. The smaller relative SHG intensities and the shift to low temperature of the maximum value in air compared to those in $\mathrm{N}_{2}$ are ascribed to oxidative degradation of the chromophores in air. There was no much difference of the relative SHG 
intensities between PI-1 and PI-2. These result are in agreement with those obtained by Inaba et al, [6] and implies that the choromophores in the poling during imidization have a similar high mobility to those in the poling after imidization above $T_{g}$ of PI.

The effect of direct current (DC) electric field on the relative SHG intensity of various PI films is shown in Figure 8. The maximum intensity was obtained under $5 \mathrm{KV}$. The higher electric field may damage the choromophores.

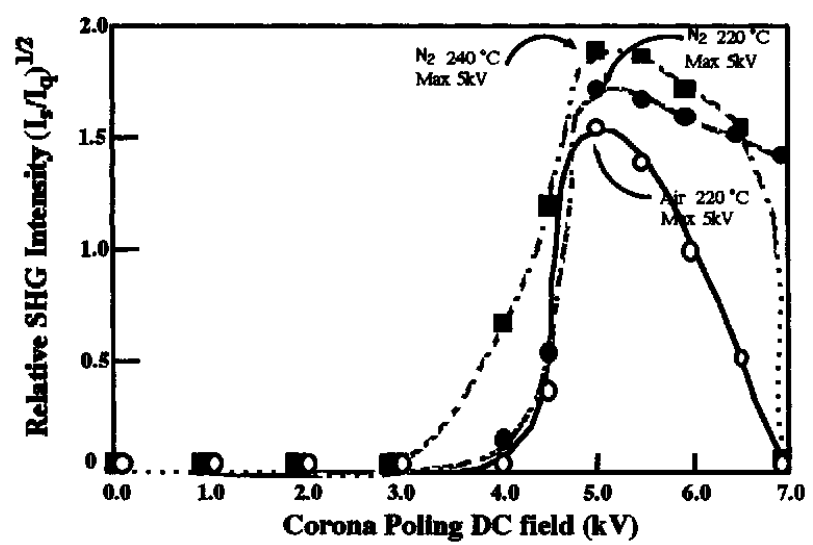

Figure 8. Effect of DC electric field on the SHG intensity of various PI films.

\subsection{Order Parameter.}

The evaluation of dipole orientation of chromophores in the polymer subjected to a DC electric field is generally carried out by using UV-visible absorption spectroscopy. The maximum absorbances due to the chromophores are reduced when the molecular dipoles are aligned along the direction of the high electric field. The effect of poling is generally expressed as an order parameter as $\Phi=1-A_{1} / A_{0}$, where $A_{0}$ and $A_{1}$ are the absorbances of the polymer film before and after corona poling.

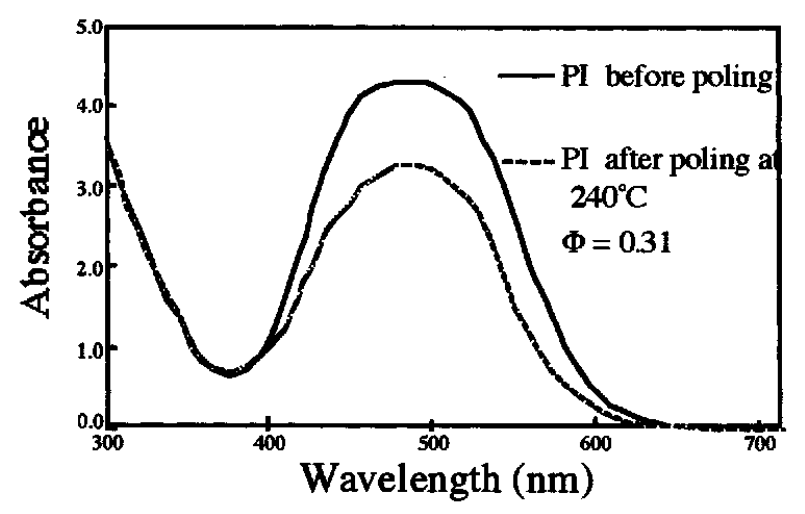

Figure 9. UV-visible spectra of PI and PI-1 before and after corona poling.
Figure 9 shows the change of UV-visible absorption spectra of the polymer. The intensity of maximum absorbance decreased after corona poling, but there was no apparent shift of maximum absorbance. The order parameters of PIs poled during imidization and after imidization were 0.31 and 0.20 , respectively.

\subsection{SHG Measurements by the Maker Fringe} Method.

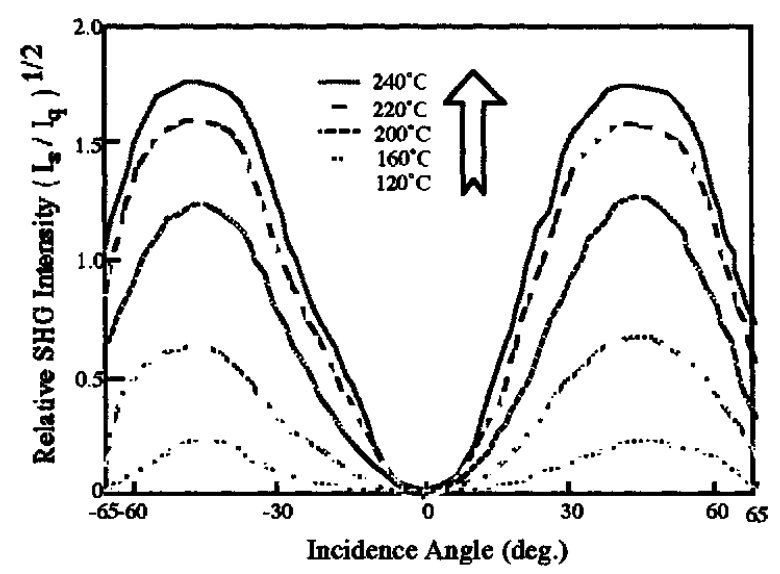

Figure 10. Relationship between the SHG intensity and the incident angle of an exciting beam for the poled PI film at various temperature.

The NLO activity of the polymer films generally determined using the second harmonic generation. The obtained Maker Fringes of the corona onset poled polymers are shown in Figure 10. The second harmonic intensity was the maximum value at an incident angle of -45 or $+45^{\circ}$. The second harmonic coefficient $\left(d_{33}\right)$ of PI-1 calculated from the angular dependence of the SHG intensity was $53 \mathrm{pm} / \mathrm{V}\left(127.3 \times 10^{-9} \mathrm{esu}\right)$.

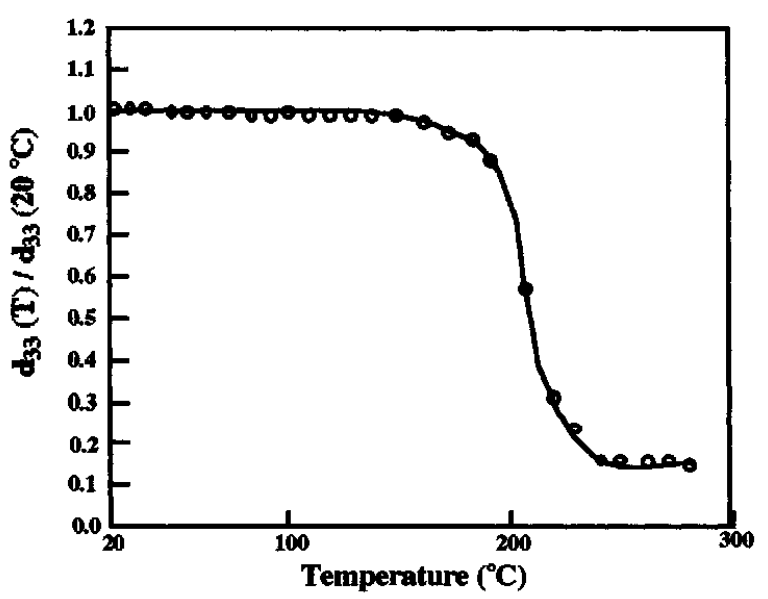

Figure 11. Relationship between the SHG stability and the temperature under $\mathrm{N}_{2}$. 
Figure 11 shows the relationship between the SHG stability and temperature under $\mathrm{N}_{2}$, where PI-1 was heated at a rate of $1{ }^{\circ} \mathrm{C} / \mathrm{min}$ from room temperature to a specified temperature, kept at this temperature for $1 \mathrm{~h}$ under $\mathrm{N}_{2}$, and measured the SHG. The SHG signal was quite stable until the temperature reached $180{ }^{\circ} \mathrm{C}$, and began to decrease rapidly at around $200{ }^{\circ} \mathrm{C}$ which is the $\mathrm{T}_{\mathrm{g}}$ of PI-1.

The studies of the isothermal SHG stability of $\mathrm{PI}-1$ at $80^{\circ} \mathrm{C}$ and $160^{\circ} \mathrm{C}$ are shown in Figure 12 and 13. At both temperatures, all of the second harmonic coefficients $\left(d_{33}\right)$ values remained almost unchanged for $360 \mathrm{~h}$. This excellent SHG stability is apparently due to the high $\mathrm{T}_{\mathrm{g}}$ of PI-1 and the need to move substantial segments of the main chain in the relaxation of the chromophores.

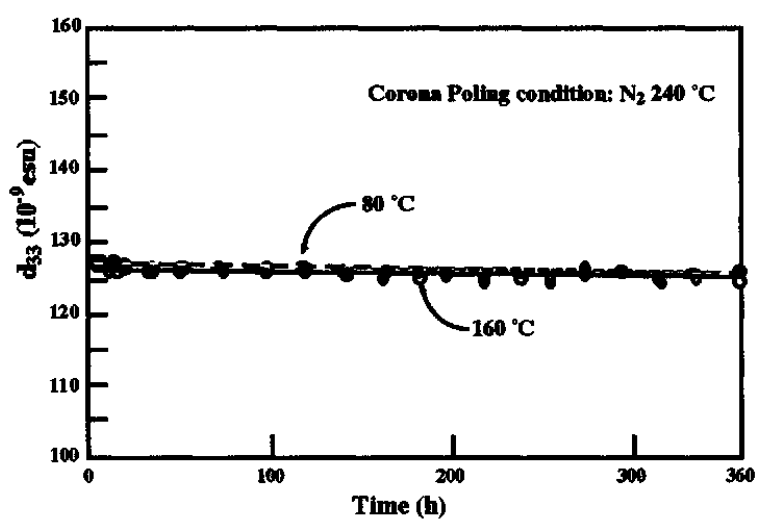

Figure 12 . Isothermal SHG stability of PI-1 at 80 and 160 ${ }^{\circ} \mathrm{C}$ under $\mathrm{N}_{2}$.

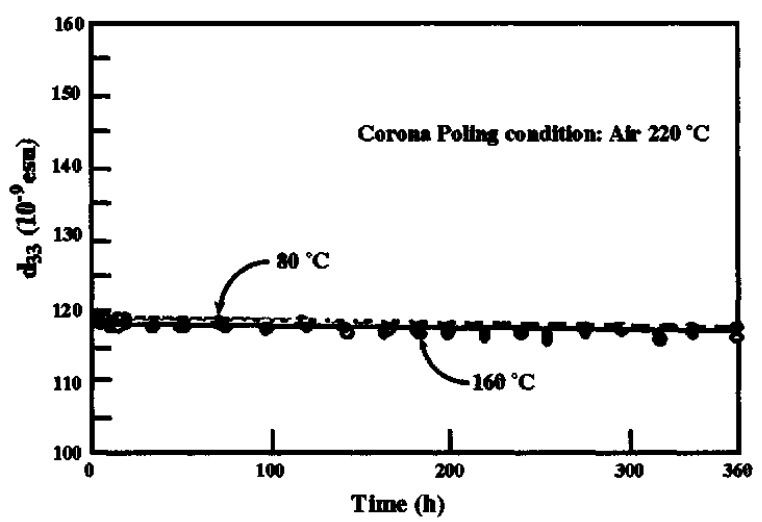

Figure 13. Isothermal SHG stability of PI-2 at 80 and 160 ${ }^{\circ} \mathrm{C}$ under air.

\section{Conclusion}

A positive-working alkaline developable photosensitive and second order nonlinear optical polyimide system has been developed, which is based on the PAA bearing NLO side chains and diazonaphtoquinone as a dissolution inhibitor. The resist gave $8 \mu \mathrm{m}$ features through UV light irradiation, followed by developing with the $0.5 \%$ aqueous TMAH solution,

The corona poling process of the PAA to the PI was studied in detail by measuring the SHG from the polymer films. The relative SHG intensities of polymers poled during and after imidization were almost same. This means that the choromophores in the poling after imidization have a similar high mobility to those in the poling during imidization above the $T_{g}$ of PI. The optimum second harmonic coefficient $\left(\mathrm{d}_{33}\right)$ of the PI was $53 \mathrm{pm} / \mathrm{N}$, evaluated from the SHG measurements using an exciting beam at $1064 \mathrm{~nm}$ and the value remained unchanged at elevated temperatures.

\section{Acknowledgement}

The authors are indebted to Mr. Sadao Kato for his technical assistance and Mr. Takeyoshi Takahashi for performing the elemental analyses.

\section{References}

1. H. Nakanishi, H. Matsuda, and S. Okada, in "Photofunctional Materials" ed by T. Tsuruta, Kyouritu Publisher, Tokyo, 1991, p.41.

2. a ) T. Verbiest, D.M. Burland, M.C. Jurich, V.Y. Lee, R.D. Miller, and w. Volksen, Science, 268 (1995) 1604. ; b) R. D. Miller, D. M. Burland, M. C. Jurich, C. R. Moylan, J. I. Thackara, R. J. Twieg, T. Verbiest and W. Volksen Macromolecules. 28, 4970 (1995). ; c) T-A. Chen, A.K-Y. Jen and Y. Cai Macromolecules, 29, 535 (1996). ; d) D.Yu, A. Gharavi, and L.Yu, J.Am.Chem.Soc. 117, 11680-11686 (1995). ; e) A.K-Y.Jen, Y.Cai, K.J.Droat, Y.J.Liu, V P.Rao, TA.Chen, R.M.Mininni, and J.T. Keenney, Polym.Mater.Sci.Eng. 72, 213 (1995).

3. T. Omote, in "Polyimides" ed by M..K. Ghosh,, and K. L. Mittal, Marcel Dekken Inc., New York (1996) p.121.

4. M. Ueda, Y. Sakai, T. Nakayama, O. Haba, Y. Ishitaka and Y. Sasaki J. Photopolymer, Sci. Technol. (1997), 10,37-42.

5. Y. Sakai, O. Haba, S. Kato, M. Ueda, T. Fukuda and H. Matsuda J. Photopolymer, Sci. Technol. (1998), 11, 217-224.

6. R.Inaba, M.Sagawa, M.Isogai, and A.Kakuta, Macromolecules, 29 (1996) 2954 